\title{
GENERALIZED AMENABILITY PROPERTIES OF THE BEURLING ALGEBRAS
}

\author{
F. GHAHRAMANI ${ }^{凶}$, E. SAMEI and YONG ZHANG
}

(Received 8 October 2010; accepted 24 December 2010)

Communicated by G. A. Willis

\begin{abstract}
We show that every one-codimensional closed two-sided ideal in a boundedly approximately contractible Banach algebra has a bounded approximate identity. We use this to give a complete characterization of bounded approximate contractibility of Beurling algebras associated to symmetric weights. We give a slight modification of a criterion for bounded approximate contractibility. We use our criterion to show that, for the quasi-SIN groups, in the presence of a certain growth condition on a weight, the associated Beurling algebra is boundedly approximately amenable if and only if it is boundedly approximately contractible. We show that approximate amenability of a Beurling algebra on an IN group necessitates the amenability of the group. Finally, we show that, for every locally compact abelian group, in the presence of a growth condition on the weight, $2 n$-weak amenability of the associated Beurling algebra is equivalent to every point-derivation vanishing at the augmentation character.
\end{abstract}

2010 Mathematics subject classification: primary 46H20; secondary 43A20.

Keywords and phrases: amenable group, Beurling algebra, bounded approximate amenability, bounded approximate contractibility, one-codimensional ideal, IN group, quasi-SIN group, $n$-weak amenability.

\section{Introduction and preliminaries}

Suppose that $G$ is a locally compact group with a fixed left Haar measure $\lambda$ (we sometimes write $d x$ or $d y$ if there is no risk of ambiguity). A Borel measurable function $\omega: G \rightarrow(0, \infty)$ is said to be a weight if it is bounded on compact subsets of $G$ and $\omega(x y) \leq \omega(x) \omega(y)$ for all $x$ and $y$ in $G$. We recall that two weights $\omega_{1}$ and $\omega_{2}$ defined on the group $G$ are equivalent if there exist $m>0$ and $M>0$ such that $m \omega_{1}(x) \leq \omega_{2}(x) \leq M \omega_{1}(x)$ for $\lambda$-almost all $x \in G$. According to [7, Remark 8.8], a weight is always equivalent to a continuous weight. Let $L^{1}(G)$ and $M(G)$ be the group algebra and the measure algebra of $G$, respectively. The Beurling algebra $L^{1}(G, \omega)$

Ghahramani was supported by NSERC Grant 36640-07, Samei was supported by NSERC Grant 366606609, Zhang was supported by NSERC Grant 238949-05.

(C) 2011 Australian Mathematical Publishing Association Inc. 1446-7887/2011 \$16.00 
associated with the group $G$ and weight $\omega$ is the space of all (equivalence classes of) functions $f$ on $G$ such that $f \omega \in L^{1}(G)$. We define the norm $\|\cdot\|_{\omega}$ and convolution product $*$ by $\|f\|_{\omega}=\|f \omega\|_{1}$ and

$$
(f * g)(x)=\int_{G} f(y) g\left(y^{-1} x\right) d y \quad \forall x \in G,
$$

for all $f, g \in L^{1}(G, \omega)$ (often when we write $\forall$ in a formula, it means 'for almost all', as here). Then $L^{1}(G, \omega)$ is a Banach algebra. Note that if the weights $\omega_{1}$ and $\omega_{2}$ are equivalent, then $L^{1}\left(G, \omega_{1}\right)$ and $L^{1}\left(G, \omega_{2}\right)$ are isomorphic as Banach algebras.

We shall also consider the weighted measure algebra $M(G, \omega)$, which is the space of all Borel measures $\mu$ such that $\omega|\mu| \in M(G)$, and

$$
\|\mu\|_{\omega}=\int_{G} \omega(t) d|\mu|(t) .
$$

The space $M(G, \omega)$ can be identified with the dual space of $C_{0}(G, 1 / \omega)$, where $f \in$ $C_{0}(G, 1 / \omega)$ if and only if $f / \omega \in C_{0}(G)$, and the norm on $C_{0}(G, 1 / \omega)$ is defined by

$$
\|f\|=\sup _{x \in G}\left|\frac{f(x)}{\omega(x)}\right|
$$

for all $f \in C_{0}(G, 1 / \omega)$. We define similarly the Banach space $L^{\infty}(G, 1 / \omega)$, so that $L^{\infty}(G, 1 / \omega)=L^{1}(G, \omega)^{*}$.

We now recall some background on approximate versions of amenability for Banach algebras. If $\mathcal{A}$ is a Banach algebra and $X$ is a Banach $\mathcal{A}$-bimodule, then a derivation $D: \mathcal{A} \rightarrow X$ is approximately inner if, for some net $\left(x_{i}\right) \subset X$,

$$
D(a)=\lim _{i} \operatorname{ad}_{x_{i}}(a) \quad \forall a \in \mathcal{A} .
$$

Here, $\operatorname{ad}_{x}$ stands for the inner derivation implemented by $x$. The Banach algebra $\mathcal{A}$ is approximately amenable if every continuous derivation $D: \mathcal{A} \rightarrow X^{*}$ is approximately inner for all dual Banach $\mathcal{A}$-bimodules $X^{*}[6]$, and it is boundedly approximately amenable if the net $\left(x_{i}^{*}\right)$ can always be chosen in such a way that the net $\left(\operatorname{ad}_{x_{i}^{*}}\right)$ is bounded in the norm of $B\left(\mathcal{A}, X^{*}\right)$. The algebra $\mathcal{A}$ is boundedly approximately contractible if the above requirements are satisfied for all continuous derivations into all Banach $\mathcal{A}$-bimodules $X$.

In [10], Grønbæk gave a complete characterization of amenability for a Beurling algebra $L^{1}(G, \omega)$. In [7], a study of various approximate versions of the notions of amenability and contractibility for Beurling algebras was initiated. As can be seen from Grønbæk's result for a weight $\omega$, the weight $\Omega$, given by $\Omega(x)=\omega(x) \omega\left(x^{-1}\right)$ for all $x \in G$, plays an important role. We call $\Omega$ the symmetrization of $\omega$.

The outstanding question is whether the three notions of amenability, bounded approximate amenability, and bounded approximate contractibility coincide for a Beurling algebra $L^{1}(G, \omega)$. It follows from the general results of this paper that, for a symmetric weight $\omega$, the Beurling algebra $L^{1}(G, \omega)$ is boundedly approximately 
contractible if and only if it is amenable and hence, in conjunction with the result of Grønbæk cited above, we have a complete characterization of bounded approximate contractibility in this case.

We recall that a locally compact group $G$ is $I N$ (for invariant neighborhood) if there is a compact neighborhood of the identity $e$ in $G$ that is invariant under all inner automorphisms of $G$, and that $G$ is $\operatorname{SIN}$ (for small invariant neighborhoods) if there is a basis of neighborhoods of the identity of $G$, each of which is invariant under the inner automorphisms of $G$ (see [13, p. 529]). The group $G$ is quasi-SIN (see [1]) if $L^{1}(G)$ has a quasi-central bounded approximate identity.

We show that if $G$ is an IN group, then approximate amenability of $L^{1}(G, \omega)$ necessitates amenability of $G$. We also show that if $G$ is a quasi-SIN group and $\omega$ is a weight such that $\lim _{x \rightarrow \infty} \omega(x) \omega\left(x^{-1}\right)=\infty$, then $L^{1}(G, \omega)$ is boundedly approximately amenable if and only if it is boundedly approximately contractible.

For $n \in \mathbb{N}$, let $\mathcal{A}^{(n)}$ denote the $n$th continuous dual of the Banach algebra $\mathcal{A}$. The space $\mathcal{A}^{(n)}$ is naturally a dual Banach $\mathcal{A}$-bimodule. The algebra $\mathcal{A}$ is $n$-weakly amenable if every continuous derivation from $\mathcal{A}$ into $\mathcal{A}^{(n)}$ is inner [3]. It was conjectured in [4] that, if $G$ is an abelian group, $\omega$ is a weight, and $\inf _{n} \omega\left(x^{n}\right) / n=0$ for all $x \in G$, then $L^{1}(G, \omega)$ is 2-weakly amenable. In [4, 8, 14], various positive partial results towards this conjecture were obtained. In the final section of this paper, we extend these results.

\section{Ideals of codimension one}

It is known that a boundedly approximately contractible Banach algebra must have a bounded approximate identity [2]. However, a complemented closed ideal of such an algebra may fail to have a bounded approximate identity [7]. Whether or not a finite codimensional closed ideal of such an algebra has a bounded approximate identity is an open question [17]. However, if the ideal has codimension one, we have the following result which turns out to be useful in studying approximate amenability of Beurling algebras. Throughout this section, for a Banach algebra $\mathcal{A}$, we do not insist that the direct sum in the unitization $\mathcal{A}^{\sharp}=\mathcal{A} \oplus \mathbb{C}$ is an $\ell^{1}$-direct sum.

THEOREM 2.1. Let $\mathcal{A}$ be a boundedly approximately contractible Banach algebra. If $I \subset \mathcal{A}$ is a closed two-sided ideal of codimension one, then I has a bounded approximate identity.

Proof. By [2, Theorem 3.3], $\mathcal{A}$ has a bounded approximate identity, $\left(e_{i}\right)$, say. Now, since $I$ is a closed ideal of codimension one, we have $I=\operatorname{ker} \phi$ for some multiplicative linear functional $\phi$ on $\mathcal{A}$. Let $\mathcal{A}^{\sharp}=\mathcal{A} \oplus \mathbb{C} e$ be the unitization of $\mathcal{A}$, where $e$ denotes the adjoined unit. We can extend $\phi$ to a multiplicative linear functional $\tilde{\phi}$ on $\mathcal{A}^{\sharp}$ by setting

$$
\tilde{\phi}(a+z e)=\phi(a)+z \quad \forall a \in \mathcal{A} \text { and } z \in \mathbb{C} .
$$

Then $J=\operatorname{ker} \tilde{\phi}$ has codimension one as a subspace of $\mathcal{A}^{\sharp}$. In fact, $\mathcal{A}^{\sharp}=J \oplus \mathbb{C} e \cong J^{\sharp}$. 
Now $\mathcal{A}^{\sharp}$ is boundedly approximately contractible, since $\mathcal{A}$ is so. Thus $J$ is boundedly approximately contractible. Hence $J$ has a bounded approximate identity, $\left(j_{\alpha}\right)$, say, (see [2]). For each $\alpha$,

$$
j_{\alpha}=a_{\alpha}+c_{\alpha} e
$$

for some $a_{\alpha} \in A$ and $c_{\alpha} \in \mathbb{C}$ such that $c_{\alpha}=-\phi\left(a_{\alpha}\right)$.

On the other hand, $\phi\left(e_{i}\right) \rightarrow 1$ since $\left(e_{i}\right)$ is a bounded approximate identity. If we set $E_{i}=\phi\left(e_{i}\right)^{-1} e_{i}$, then $\left(E_{i}\right)$ is still a bounded approximate identity for $\mathcal{A}$ and $\phi\left(E_{i}\right)=1$ for all $i$.

Let

$$
b_{\alpha, i}=a_{\alpha}+c_{\alpha} E_{i} \text {. }
$$

Then $\left(b_{\alpha, i}\right) \subset I$ is a bounded net. We know that $j_{\alpha} f \rightarrow f$ and $f j_{\alpha} \rightarrow f$ for all $f \in I$. Thus

$$
b_{\alpha, i} f=j_{\alpha} f+\left(c_{\alpha} E_{i} f-c_{\alpha} f\right)=j_{\alpha} f+c_{\alpha}\left(E_{i} f-f\right) \rightarrow f,
$$

and similarly

$$
f b_{\alpha, i} \rightarrow f
$$

Therefore $\left(b_{\alpha, i}\right)$ is a bounded approximate identity for $I$.

COROLlary 2.2. Suppose that $\omega$ is a weight on the locally compact group $G$ and define $\Omega(t)=\omega(t) \omega\left(t^{-1}\right)$ for all $t \in G$. Then the following conditions are equivalent.

(1) The Beurling algebra $L^{1}(G, \Omega)$ is boundedly approximately contractible.

(2) The Beurling algebra $L^{1}(G, \omega)$ is amenable.

(3) The group $G$ is amenable and $\Omega$ is bounded on $G$.

PROOF. In light of [10, Theorem 0], it suffices to show that (1) implies (2). Let us assume that $L^{1}(G, \Omega)$ is boundedly approximately contractible and suppose that $I_{0}$ is the augmentation ideal in $L^{1}(G, \Omega)$, that is

$$
I_{0}=\left\{f \in L^{1}(G, \Omega): \int_{G} f(x) d x=0\right\} .
$$

Since $I_{0}$ is of codimension one and $L^{1}(G, \Omega)$ is boundedly approximately contractible, Theorem 2.1 implies that $I_{0}$ has a bounded approximate identity, $\left(f_{\beta}\right)$, say.

Let $\phi \in L^{1}(G, \Omega)$ be such that $\int_{G} \phi(x) d x=1$. Then $\delta_{x} * \phi-\phi \in I_{0}$ for all $x \in G$, whence

$$
\delta_{x} * \phi * f_{\beta}-\phi * f_{\beta}-\delta_{x} * \phi+\phi \rightarrow 0 .
$$

Equivalently,

$$
\delta_{x} *\left(\phi-\phi * f_{\beta}\right)-\left(\phi-\phi * f_{\beta}\right) \rightarrow 0 .
$$

Since $\left\langle\phi-\phi * f_{\beta}, 1\right\rangle=1$, condition (c) of [10, Theorem 0$]$ holds and so $L^{1}(G, \omega)$ is amenable.

Recall that a weight $\omega$ on the group $G$ is symmetric if $\omega\left(x^{-1}\right)=\omega(x)$ for all $x \in G$. 
COROLlary 2.3. Let $\omega$ be a symmetric weight on $G$. Then $L^{1}(G, \omega)$ is boundedly approximately contractible if and only if it is amenable.

Proof. Suppose that $L^{1}(G, \omega)$ is boundedly approximately contractible. Consider the weight $\omega^{\prime}=\sqrt{\omega}$ on $G$. Then $\omega=\Omega^{\prime}$, the symmetrization of $\omega^{\prime}$. So, by Corollary 2.2, $L^{1}\left(G, \omega^{\prime}\right)$ is amenable. It now follows by [10, Theorem 0] that $L^{1}\left(G, \Omega^{\prime}\right)=L^{1}(G, \omega)$ is amenable.

The converse is trivial: every amenable Banach algebra is automatically boundedly approximately contractible (see the note following [7, Definition 5.1]).

We do not know whether the conclusion of Theorem 2.1 holds if we only assume that $\mathcal{A}$ is boundedly approximately amenable. If this is true, then the above two corollaries would hold with 'boundedly approximately contractible' replaced by 'boundedly approximately amenable'. We can show that this is the case under some additional conditions. First, we note that the argument of [2, Theorem 3.3] works verbatim to prove the following lemma.

LEMMA 2.4. Suppose that $\mathcal{A}$ is a boundedly approximately amenable Banach algebra. Let $\left(a_{\alpha}\right),\left(b_{\beta}\right)$ be two nets of elements of $\mathcal{A}$. If

$$
\lim _{\alpha} b_{\beta} a_{\alpha}=b_{\beta} \forall \beta \quad \text { and } \quad \lim _{\beta} b_{\beta} a_{\alpha}=a_{\alpha} \forall \alpha,
$$

and there exists $K>0$ such that $\left\|a a_{\alpha}\right\| \leq K\|a\|$ and $\left\|b_{\beta} a\right\| \leq K\|a\|$ for all $a \in \mathcal{A}$ and all $\alpha$ and $\beta$, then $\left(a_{\alpha}\right)$ and $\left(b_{\beta}\right)$ are bounded.

As in the proof of Theorem 2.1, given a one-codimensional closed ideal $I$ of $\mathcal{A}$, we may define

$$
J=\{a+t e: a \in \mathcal{A}, t \in \mathbb{C}, \phi(a)+t=0\},
$$

where $\phi$ is a multiplicative linear functional on $\mathcal{A}$ for which $I=\operatorname{ker} \phi$. Then $J$ is a subalgebra of $\mathcal{A}^{\sharp}$ and $I$ is a closed ideal of $J$; further, $I=\mathcal{A} J=J \mathcal{A}=\mathcal{A} J \mathcal{A}$ and $J^{\sharp} \cong \mathcal{A}^{\sharp}=\mathcal{A} \oplus \mathbb{C} e$.

PROPOSITION 2.5. Suppose that $\mathcal{A}$ is a boundedly approximately amenable Banach algebra with a bounded approximate identity, and that I is a closed codimension-one ideal of $\mathcal{A}$. Suppose that $J$ is the closed ideal of $\mathcal{A}^{\sharp}$ associated to I as defined above. Furthermore, suppose that the following conditions hold.

(1) There is a dual Banach left $\mathcal{A}$-module $M_{l}$ such that, as a left $\mathcal{A}$-module, J may be continuously embedded into $M_{l}$, and $I=\mathcal{A} M_{l}$ after this embedding.

(2) There is a dual Banach right $\mathcal{A}$-module $M_{r}$ such that, as a right $\mathcal{A}$-module, $J$ may be continuously embedded into $M_{r}$, and $I=M_{r} \mathcal{A}$ after this embedding.

Then I has a bounded approximate identity.

PROOF. If $\mathcal{A}$ is boundedly approximately amenable, then so is $J$, by the argument used in the proof of Theorem 2.1. The module $M_{l}$ is naturally extended to a dual left $\mathcal{A}^{\sharp}$-module and, hence, may be regarded as a Banach dual left $J$-module. We define 
a trivial right module action on $M_{l}$ so that the embedding map $\tau: J \rightarrow M_{l}$ becomes a continuous derivation from $J$ into $M_{l}$.

Since $J$ is boundedly approximately amenable and $M_{l}$ is a dual Banach $J$-bimodule, there exist a net $\left(m_{i}\right) \subset M_{l}$ and a positive constant $K$ such that $a m_{i} \rightarrow a$ and $\left\|a m_{i}\right\| \leq K\|a\|$ for all $a \in J$. Let $\left(e_{j}\right) \subset \mathcal{A}$ be a bounded approximate identity for $\mathcal{A}$. We define a net by setting $a_{\alpha}=e_{j} m_{i}$, where $\alpha=(i, j)$ is given the product order direction. Then $\left(a_{\alpha}\right) \subset I$ by the hypothesis.

Moreover, $\left\|a a_{\alpha}\right\| \leq c K\|a\|$ for all $a \in J$ and all $\alpha$, where $c$ is a bound for $\left(e_{j}\right)$. Note that $\lim _{j} a e_{j}=a$ and $\lim _{i} a m_{i}=a$. Therefore

$$
\begin{aligned}
\left\|a a_{\alpha}-a\right\| & \leq\left\|a e_{j} m_{i}-a m_{i}\right\|+\left\|a m_{i}-a\right\| \\
& \leq K\left\|a e_{j}-a\right\|+\left\|a m_{i}-a\right\| \rightarrow 0,
\end{aligned}
$$

for all $a \in I$.

Now, working with $M_{r}$ similarly, we find a net $\left(b_{\beta}\right) \subset I$ such that $\left\|b_{\beta} a\right\| \leq c K\|a\|$ for all $a \in J$ and all $\beta$, and $\lim _{\beta} b_{\beta} a=a$ for all $a \in I$. Applying Lemma 2.4 for $J$, we deduce that $\left(a_{\alpha}\right)$ and $\left(b_{\beta}\right)$ are bounded. So they are bounded right and bounded left approximate identities for $I$, respectively. This implies that $I$ has a bounded approximate identity.

Corollary 2.6. Suppose that the Banach algebra $\mathcal{A}$ is boundedly approximately amenable and has a bounded approximate identity. If the operators of left and right multiplication by elements of $\mathcal{A}$ are weakly compact on $\mathcal{A}$, then every closed two-sided ideal of codimension one in $\mathcal{A}$ has a bounded approximate identity.

PROOF. Let $I$ be a closed two-sided ideal of codimension one of $\mathcal{A}$ and let $J$ be the ideal associated to $I$ as in the proof of Theorem 2.1. Since $\mathcal{A}$ has a bounded approximate identity, the Cohen factorization theorem implies that $I=I \mathcal{A}=\mathcal{A} I$.

Now we take $M_{l}=M_{r}=J^{* *}$. By the hypothesis, $\mathcal{A}$ is a two-sided ideal in $\mathcal{A}^{* *}$. Hence

$$
\mathcal{A} J^{* *} \subseteq \mathcal{A} \mathcal{A}^{* *}+\mathcal{A} \subseteq \mathcal{A} .
$$

Now let $\phi$ be the multiplicative linear functional whose kernel is $I$. Then, since $\phi$ vanishes on $\mathcal{A} J^{* *}$, we have $\mathcal{A} J^{* *} \subseteq I$. We also have

$$
\mathcal{A} J^{* *} \supseteq \mathcal{A} J \supseteq \mathcal{A} I=I .
$$

Hence $\mathcal{A} J^{* *}=I$. We can similarly prove that $J^{* *} \mathcal{A}=I$, and our result follows by Proposition 2.5.

COROLlary 2.7. Let $\omega$ be a weight on $G$ such that $\lim _{x \rightarrow \infty} \omega(x)=\infty$. If $L^{1}(G, \omega)$ is boundedly approximately amenable, then the augmentation ideal

$$
I_{0}=\left\{f \in L^{1}(G, \omega): \int_{G} f(x) d x=0\right\}
$$

has a bounded approximate identity. 
PROOF. Under the given condition, $\omega$ is bounded away from 0 . Thus, $I_{0}$ is a closed ideal of $L^{1}(G, \omega)$ with codimension one. The space $M(G, \omega)$ is a dual Banach $L^{1}(G, \omega)$-module with predual module $C_{0}\left(G, \omega^{-1}\right)$. Since $1 \in C_{0}\left(G, \omega^{-1}\right)$,

$$
M_{0}=\{m \in M(G, \omega):\langle 1, m\rangle=0\}
$$

is a weak* closed submodule of $M(G, \omega)$.

Let $M_{l}=M_{r}=M_{0}$. Then conditions (1) and (2) of Proposition 2.5 are satisfied when

$$
J=J_{0}=\left\{f+t \delta_{e}: f \in L^{1}(G, \omega), t \in \mathbb{C}, \int_{G} f(x) d x+t=0\right\} .
$$

The result now follows immediately by Proposition 2.5 .

Corollary 2.8. Let $\omega$ be a symmetric weight on $G$. If $\lim _{x \rightarrow \infty} \omega(x)=\infty$, then $L^{1}(G, \omega)$ is not boundedly approximately amenable.

Proof. Let $\omega^{\prime}=\sqrt{\omega}$. Then $\omega=\Omega^{\prime}$, the symmetrization of $\omega^{\prime}$. Suppose that $\lim _{x \rightarrow \infty} \omega(x)=\infty$ and $L^{1}(G, \omega)$ is boundedly approximately amenable. Then the augmentation ideal of $L^{1}(G, \omega)$ has a bounded approximate identity by Corollary 2.7. The argument of Corollary 2.2 implies that $L^{1}\left(G, \omega^{\prime}\right)$ is amenable and, hence, $\Omega^{\prime}=\omega$ is bounded, a contradiction.

EXAMPLE 2.9. If a continuous weight $\omega$ on $G$ satisfies

$$
\limsup _{x \in G} \frac{\omega\left(x^{-1}\right)}{\omega(x)} \leq L,
$$

for some constant $L>0$, and $\lim _{x \rightarrow \infty} \omega(x)=\infty$, then $L^{1}(G, \omega)$ is not boundedly approximately amenable.

PROOF. The condition implies that there are positive constants $C$ and $c$ such that

$$
c \omega\left(x^{-1}\right) \leq \omega(x) \leq C \omega\left(x^{-1}\right) \quad \forall x \in G .
$$

So

$$
\sqrt{c} \omega^{\prime}(x) \leq \omega(x) \leq \sqrt{C} \omega^{\prime}(x) \quad \forall x \in G,
$$

and $\omega^{\prime}(x)=\sqrt{\Omega(x)}$ is symmetric on $G$. By Corollary $2.8, L^{1}\left(G, \omega^{\prime}\right) \cong L^{1}(G, \omega)$ are not boundedly approximately amenable.

EXAMPLE 2.10. The preceding corollary covers a large class of weights that are defined on compactly generated locally compact groups. More precisely, suppose that $G$ is noncompact and $U$ is a compact neighborhood of the identity that generates $G$. By replacing $U$ with $U \cap U^{-1}$, we may assume that $U^{-1}=U$. Now define the function $\tau: G \rightarrow \mathbb{N}$ by

$$
\tau(x)=\min \left\{n \in \mathbb{N}: x \in U^{n}\right\} \quad \forall x \in G .
$$

It is straightforward to verify that $\tau$ is Borel measurable, symmetric and locally bounded. 
Moreover, $\tau(x y) \leq \tau(x)+\tau(y)$ for all $x, y \in G$. Thus, when $\alpha>0$ and $0<\beta \leq 1$, the maps $\omega_{\alpha}, \sigma_{\beta}: G \rightarrow(0, \infty)$, given by

$$
\omega_{\alpha}(x)=(1+\tau(x))^{\alpha} \quad \text { and } \quad \sigma_{\beta}(x)=e^{\tau(x)^{\beta}},
$$

are symmetric weights on $G$. In addition, since $G$ is not compact,

$$
\lim _{x \rightarrow \infty} \omega_{\alpha}(x)=\lim _{x \rightarrow \infty} \sigma_{\beta}(x)=\infty .
$$

Therefore, by Corollary 2.8, neither $L^{1}\left(G, \omega_{\alpha}\right)$ nor $L^{1}\left(G, \sigma_{\beta}\right)$ is boundedly approximately amenable.

The weights $\omega_{\alpha}$ and $\sigma_{\beta}$ are called polynomial and exponential weights on $G$, respectively. In the special cases where $G=\mathbb{Z}$ or $G=\mathbb{R}$, these are just the classical polynomial and exponential weights.

\section{A characterization of bounded approximate contractibility}

In [2, 7] various characterizations of bounded approximate amenability and bounded approximate contractibility are given. Below we give a slightly different characterization of bounded approximate contractibility that is more suited to our purposes in this paper than those obtained previously.

In the following proposition, we call a net $\left(u_{i}\right) \subset \mathcal{A} \hat{\otimes} \mathcal{A}$ a multiplier-bounded approximate diagonal if there exists a constant $C>0$ such that

$$
\begin{array}{r}
a \cdot u_{i}-u_{i} \cdot a \rightarrow 0 \quad\left\|a \cdot u_{i}-u_{i} \cdot a\right\| \leq C\|a\| \\
\pi\left(u_{i}\right) a \rightarrow a \quad\left\|\pi\left(u_{i}\right) a\right\| \leq C\|a\|
\end{array}
$$

for all $a \in \mathcal{A}$ and all $i$.

Proposition 3.1. Let $\mathcal{A}$ be a Banach algebra. Then the following conditions are equivalent.

(1) The Banach algebra $\mathcal{A}$ is boundedly approximately contractible.

(2) The Banach algebra $\mathcal{A}$ has a bounded approximate identity and has a multiplierbounded approximate diagonal.

(3) There exists a multiplier-bounded approximate diagonal $\left(u_{i}\right) \subset \mathcal{A} \hat{\otimes} \mathcal{A}$ such that $\left(\pi\left(u_{i}\right)\right)$ is bounded.

Proof. We first show that (3) implies (1). Suppose that $\left(u_{i}\right)$ is a multiplier-bounded approximate diagonal and $\left(\pi\left(u_{i}\right)\right)$ is bounded. Then we let

$$
m_{(i, j)}=u_{i}+\left(e-\pi\left(u_{i}\right)\right) \otimes\left(e-\pi\left(u_{j}\right)\right),
$$

where $e$ is the identity of $\mathcal{A}^{\sharp}$. Now $m_{(i, j)} \in \mathcal{A}^{\sharp} \hat{\otimes} \mathcal{A}^{\sharp}$ and there is a constant $C>0$ such that $\left\|f \cdot m_{(i, j)}-m_{(i, j)} \cdot f\right\| \leq C\|f\|$ for all $f \in \mathcal{A}^{\sharp}$ and all $i$ and $j$.

Also, $\pi\left(m_{(i, j)}\right)=e+\pi\left(u_{i}\right) \cdot \pi\left(u_{j}\right)-\pi\left(u_{j}\right)$. So $\left(\pi\left(m_{(i, j)}\right)\right)$ is a bounded net with product order direction. It is easily seen that there is a subnet $\left(m_{\alpha}\right)$ of $\left(m_{(i, j)}\right)$ 
such that $\left\|a \cdot m_{\alpha}-m_{\alpha} \cdot a\right\| \rightarrow 0$ for all $a \in \mathcal{A}$ and $\pi\left(m_{\alpha}\right) \rightarrow e$. Thus $\mathcal{A}^{\sharp}$ has a multiplier-bounded approximate diagonal. By [2, Proposition 2.2], $\mathcal{A}$ is boundedly approximately contractible.

Now we prove that (1) implies (2). If $\mathcal{A}$ is boundedly approximately contractible, then $\mathcal{A}$ has a bounded approximate identity, say $\left(e_{\alpha}\right)$ (see [2, Corollary 3.4]). Let $\left(m_{\alpha}\right) \subset \mathcal{A}^{\sharp} \hat{\otimes} \mathcal{A}^{\sharp}$ be a multiplier-bounded approximate diagonal for $\mathcal{A}^{\sharp}$. We may assume that $\pi\left(m_{\alpha}\right)=e$, and so we can write

$$
m_{\alpha}=M_{\alpha}-F_{\alpha} \otimes e-e \otimes G_{\alpha}+e \otimes e,
$$

where $M_{\alpha} \in \mathcal{A} \hat{\otimes} \mathcal{A}$ and $F_{\alpha}, G_{\alpha} \in \mathcal{A}$.

Now $a \cdot m_{\alpha}-m_{\alpha} \cdot a \rightarrow 0$ and there exists $C>0$ such that

$$
\left\|a \cdot m_{\alpha}-m_{\alpha} \cdot a\right\| \leq C\|a\| \quad \forall \alpha
$$

for all $a \in \mathcal{A}$. Thus

$$
\begin{gathered}
\left\|a \cdot M_{\alpha}-M_{\alpha} \cdot a+F_{\alpha} \otimes a-a \otimes G_{\alpha}\right\| \rightarrow 0 \\
a F_{\alpha} \rightarrow a, \quad G_{\alpha} a \rightarrow a,
\end{gathered}
$$

and there is a positive constant $C^{\prime}$ such that

$$
\begin{gathered}
\left\|a \cdot M_{\alpha}-M_{\alpha} \cdot a+F_{\alpha} \otimes a-a \otimes G_{\alpha}\right\| \leq C^{\prime}\|a\| \\
\left\|a F_{\alpha}\right\| \leq C^{\prime}\|a\|, \quad\left\|G_{\alpha} a\right\| \leq C^{\prime}\|a\|,
\end{gathered}
$$

for all $\alpha$ and $a \in \mathcal{A}$. Clearly, $\left(F_{\alpha}\right)$ and $\left(G_{\alpha}\right)$ are multiplier-bounded right and left approximate identities of $\mathcal{A}$, respectively. Since $\mathcal{A}$ has a bounded approximate identity, they must be bounded.

Now we define a net $\left(u_{(\alpha, \beta)}\right) \subset \mathcal{A} \hat{\otimes} \mathcal{A}$ by

$$
u_{(\alpha, \beta)}=M_{\alpha}-F_{\alpha} \otimes e_{\beta}-e_{\beta} \otimes G_{\alpha}+e_{\beta} \otimes e_{\beta} .
$$

Then it is easy to check that a subnet of $\left(u_{(\alpha, \beta)}\right)$ gives a multiplier-bounded approximate diagonal for $\mathcal{A}$. So (1) implies (2).

Finally, if (2) holds and $\left(u_{\alpha}\right)$ is a multiplier-bounded approximate diagonal for $\mathcal{A}$, then $\left(\pi\left(u_{\alpha}\right)\right)$ is a multiplier-bounded approximate identity for $\mathcal{A}$. It must be bounded since $\mathcal{A}$ has a bounded approximate identity by the assumption. So (3) holds.

COROLlary 3.2. Let $\mathcal{A}$ be boundedly approximately contractible, and let $J$ be a left (or right) closed ideal of $\mathcal{A}$. If $J$ is complemented in $\mathcal{A}$, then $J$ has a multiplierbounded right (or left) approximate identity. That is, there exist a net $\left(p_{i}\right) \subset J$ and a constant $c$ such that $r p_{i} \rightarrow r$ and $\left\|r p_{i}\right\| \leq c\|r\|$ (or $p_{i} r \rightarrow r$ and $\left\|p_{i} r\right\| \leq c\|r\|$, respectively) for all $r \in J$.

PROOF. We prove the result for left ideals. The proof for right ideals is similar. Let $\left(u_{i}\right) \subset \mathcal{A} \hat{\otimes} \mathcal{A}$ be a multiplier-bounded approximate diagonal for $\mathcal{A}$ such that $\left(\pi\left(u_{i}\right)\right)$ 
is bounded. Let $P: \mathcal{A} \rightarrow J$ be a continuous projection. Define $\Psi: \mathcal{A} \hat{\otimes} \mathcal{A} \rightarrow J$ by $\Psi(a \otimes b)=a P(b)$ for all $a, b \in \mathcal{A}$. Then

$$
r \Psi\left(u_{i}\right)=\pi\left(u_{i}\right) r+\Psi\left(r u_{i}-u_{i} r\right)
$$

for all $r \in J$. Let $p_{i}=\Psi\left(u_{i}\right)$. The identity above implies that $\left(p_{i}\right) \subset J$ is a multiplierbounded right approximate identity for $J$.

\section{Beurling algebras on quasi-SIN groups}

The natural right and left module actions of $L^{1}(G, \omega)$ on the space

$$
L^{1}(G, \omega) \hat{\otimes} L^{1}(G, \omega)=L^{1}(G \times G, \omega \times \omega)
$$

dualize to left and right actions of $L^{1}(G, \omega)$ on $L^{\infty}(G \times G, 1 /(\omega \times \omega))$, respectively. The space $C_{0}(G \times G, 1 /(\omega \times \omega))$ is a submodule of $L^{\infty}(G \times G, 1 /(\omega \times \omega))$ for the dualized actions, which are given by the following formulas:

$$
f \cdot \Phi(x, y)=\int_{G} \Phi(x, y \xi) f(\xi) d \xi, \quad \Phi \cdot f(x, y)=\int_{G} \Phi(\xi x, y) f(\xi) d \xi,
$$

for all $f \in L^{1}(G, \omega), \Phi \in C_{0}(G \times G, 1 /(\omega \times \omega))$ and $x, y \in G$. We define the Banach space automorphism $T$ on $C_{0}(G \times G, 1 /(\omega \times \omega))$ by

$$
T(\Phi)(x, y)=\Phi(y, x) \quad \forall x, y \in G
$$

for all $\Phi \in C_{0}(G \times G, 1 /(\omega \times \omega))$. Now, for all such $\Phi$ and $f \in L^{1}(G, \omega)$, define

$$
f \circ \Phi=T(f \cdot T(\Phi)) \quad \text { and } \quad \Phi \circ f=T(T(\Phi) \cdot f) .
$$

These formulas define Banach $L^{1}(G, \omega)$-bimodule actions on $C_{0}(G \times G, 1 /(\omega \times \omega))$. The module actions . and $\circ$ satisfy the following mixed associativity and commutativity laws for $f, g \in L^{1}(G, \omega)$, and $\Phi \in C_{0}(G \times G, 1 /(\omega \times \omega))$ :

$$
(f \cdot \Phi) \circ g=f \cdot(\Phi \circ g) \quad(f \circ \Phi) \cdot g=f \circ(\Phi \cdot g) \quad f \cdot(g \circ \Phi)=g \circ(f \cdot \Phi) .
$$

The dual module actions of $\circ$ on $C_{0}(G \times G, 1 /(\omega \times \omega))^{*}=M(G \times G, \omega \times \omega)$ are given by

$$
f \circ m=T^{*}\left(f \cdot T^{*}(m)\right) \quad \text { and } \quad m \circ f=T^{*}\left(T^{*}(m) \cdot f\right)
$$

for all $f \in L^{1}(G, \omega)$ and $m \in M(G \times G, \omega \times \omega)$. Integral formulas for the two different module actions are as follows:

$$
\begin{aligned}
& \langle\Phi, f \cdot m\rangle=\langle\Phi \cdot f, m\rangle=\int_{G^{3}} \Phi(\xi x, y) f(\xi) d \xi d m(x, y), \\
& \langle\Phi, m \cdot f\rangle=\langle f \cdot \Phi, m\rangle=\int_{G^{3}} \Phi(x, y \xi) f(\xi) d \xi d m(x, y), \\
& \langle\Phi, f \circ m\rangle=\langle\Phi \circ f, m\rangle=\int_{G^{3}} \Phi(x, \xi y) f(\xi) d \xi d m(x, y), \\
& \langle\Phi, m \circ f\rangle=\langle f \circ \Phi, m\rangle=\int_{G^{3}} \Phi(x \xi, y) f(\xi) d \xi d m(x, y) .
\end{aligned}
$$


We recall that the group $G$ is quasi-SIN if $L^{1}(G)$ has a quasi-central bounded approximate identity, namely, if there is a bounded approximate identity $\left(e_{\alpha}\right)$ for $L^{1}(G)$ such that

$$
\left\|\delta_{x} \cdot e_{\alpha}-e_{\alpha} \cdot \delta_{x}\right\| \rightarrow 0
$$

for all $x \in G$. Typical examples of quasi-SIN groups are SIN groups [12] and amenable groups [11]. Additional features of quasi-SIN groups may be seen in [15].

LEMMA 4.1. Suppose that $G$ is a quasi-SIN locally compact group and $\omega$ is a weight on $G$ satisfying $\omega(e)=1$. Then $L^{1}(G, \omega)$ has a quasi-central bounded approximate identity $\left(e_{\alpha}\right)$ such that

$$
\lim _{\alpha}\left(e_{\alpha} \cdot m \cdot e_{\alpha}-e_{\alpha} \circ m \circ e_{\alpha}\right)=0
$$

for all $m \in M(G \times G, \omega \times \omega)$.

PRoOF. If $G$ is quasi-SIN, then by [15, Theorem 2.6], $L^{1}(G)$ has a quasi-central bounded approximate identity $\left(e_{\alpha}\right)$ such that

$$
\left\|\delta_{x} \cdot e_{\alpha}-e_{\alpha} \cdot \delta_{x}\right\|_{1} \rightarrow 0
$$

the limit being uniform for $x$ in compact subsets of $G$, and $\operatorname{supp}\left(e_{\alpha}\right) \stackrel{\alpha}{\longrightarrow}\{e\}$. Since $\omega(e)=1$, the net $\left(e_{\alpha}\right)$ is also a bounded approximate identity for $L^{1}(G, \omega)$ and

$$
\left\|\delta_{x} \cdot e_{\alpha}-e_{\alpha} \cdot \delta_{x}\right\|_{\omega} \rightarrow 0
$$

uniformly for $x$ on compact sets of $G$. Let $\left\|e_{\alpha}\right\|_{\omega} \leq c$ for all $\alpha$. Since Borel measures with compact support are dense in $M(G \times G, \omega \times \omega)$, in order to prove our assertion it suffices to show that (4.1) holds for each $m$ with compact support. Let $K=\operatorname{supp}(m)$ be compact. We take a compact set $H \subset G$ such that $K \subset H^{\circ}$. Then there is a net $\left(m_{i}\right)$ in $\ell^{1}(G \times G, \omega \times \omega)$ such that $\operatorname{supp}\left(m_{i}\right) \subset H,\left\|m_{i}\right\|_{\omega \times \omega} \leq\|m\|_{\omega \times \omega}$ and $\lim _{i} m_{i}=m$ in the weak* topology. Note that, for each point mass $\delta_{(x, y)} \in \ell^{1}(G \times G, \omega \times \omega)$,

$$
f \cdot \delta_{(x, y)}-\delta_{(x, y)} \circ f=\left(f \cdot \delta_{x}-\delta_{x} \cdot f\right) \otimes \delta_{y}
$$

and

$$
f \circ \delta_{(x, y)}-\delta_{(x, y)} \cdot f=\delta_{x} \otimes\left(f \cdot \delta_{y}-\delta_{y} \cdot f\right),
$$

for all $f \in L^{1}(G, \omega)$. Thus, for all $\Phi \in C_{0}\left(G \times G, \omega^{-1} \times \omega^{-1}\right)$ such that $\|\Phi\| \leq 1$,

$$
\begin{aligned}
&\left|\left\langle\Phi,\left(e_{\alpha} \cdot m \cdot e_{\alpha}-e_{\alpha} \circ m \circ e_{\alpha}\right)\right\rangle\right| \\
& \leq\left|\left\langle\Phi, e_{\alpha} \cdot\left(m-m_{i}\right) \cdot e_{\alpha}\right\rangle\right|+\left|\left\langle\Phi, e_{\alpha} \circ\left(m-m_{i}\right) \circ e_{\alpha}\right\rangle\right| \\
&+\left\|\left(e_{\alpha} \cdot m_{i}-m_{i} \circ e_{\alpha}\right) \cdot e_{\alpha}\right\|+\left\|\left(e_{\alpha} \circ m_{i}-m_{i} \cdot e_{\alpha}\right) \circ e_{\alpha}\right\| \\
& \leq\left|\left\langle e_{\alpha} \cdot \Phi \cdot e_{\alpha},\left(m-m_{i}\right)\right\rangle\right|+\left|\left\langle e_{\alpha} \circ \Phi \circ e_{\alpha},\left(m-m_{i}\right)\right\rangle\right| \\
&+c\|m\| \sup _{(x, y) \in H}\left(\left\|e_{\alpha} \cdot \delta_{x}-\delta_{x} \cdot e_{\alpha}\right\|_{\omega}\left\|\delta_{y}\right\|_{\omega}\right) \\
&+c\|m\| \sup _{(x, y) \in H}\left(\left\|\delta_{x}\right\|_{\omega}\left\|e_{\alpha} \cdot \delta_{y}-\delta_{y} \cdot e_{\alpha}\right\|_{\omega}\right) .
\end{aligned}
$$


Since $\lim _{i} m_{i}=m$ in the weak* topology,

$$
\begin{aligned}
\left|\left\langle\Phi, e_{\alpha} \cdot m \cdot e_{\alpha}-e_{\alpha} \circ m \circ e_{\alpha}\right\rangle\right| \leq c\|m\| & \sup _{(x, y) \in H}\left\|e_{\alpha} \cdot \delta_{x}-\delta_{x} \cdot e_{\alpha}\right\|_{\omega}\left\|\delta_{y}\right\|_{\omega} \\
& +c\|m\| \sup _{(x, y) \in H}\left\|\delta_{x}\right\|_{\omega}\left\|e_{\alpha} \cdot \delta_{y}-\delta_{y} \cdot e_{\alpha}\right\|_{\omega},
\end{aligned}
$$

for all $\Phi \in C_{0}\left(G \times G, \omega^{-1} \times \omega^{-1}\right)$ satisfying $\|\Phi\| \leq 1$. Therefore

$$
\begin{aligned}
\left\|e_{\alpha} \cdot m \cdot e_{\alpha}-e_{\alpha} \circ m \circ e_{\alpha}\right\|_{\omega \times \omega} \leq c\|m\| & \sup _{(x, y) \in H}\left\|e_{\alpha} \cdot \delta_{x}-\delta_{x} \cdot e_{\alpha}\right\|_{\omega}\left\|\delta_{y}\right\|_{\omega} \\
& +c\|m\| \sup _{(x, y) \in H}\left\|\delta_{x}\right\|_{\omega}\left\|e_{\alpha} \cdot \delta_{y}-\delta_{y} \cdot e_{\alpha}\right\|_{\omega} .
\end{aligned}
$$

Now (4.1) follows by compactness of $H$ and the properties of $\left(e_{\alpha}\right)$ stated earlier in the proof.

Proposition 4.2. Suppose that $G$ is a quasi-SIN locally compact group and $\omega$ is a weight on $G$ such that $\omega(e)=1$ and

$$
\lim _{x \rightarrow \infty} \omega(x) \omega\left(x^{-1}\right)=\infty .
$$

If $L^{1}(G, \omega)$ is boundedly approximately amenable, then it is boundedly approximately contractible.

Proof. Define the $L^{1}(G, \omega)$-bimodule morphism $\tilde{\pi}: M(G \times G, \omega \times \omega) \rightarrow M(G, \omega)$ by

$$
\langle\Phi, \tilde{\pi}(u)\rangle=\int_{G^{2}} \Phi(x y) d u(x, y)
$$

for all $\Phi \in C_{0}(G, 1 / \omega)$ and $u \in M(G \times G, \omega \times \omega)$. Since $\lim _{x \rightarrow \infty} \omega(x) \omega\left(x^{-1}\right)=$ $\infty$, it follows from [7, Lemma 8.3] that $\tilde{\pi}$ is weak* continuous and is an extension of the product mapping

$$
\pi: L^{1}(G \times G, \omega \times \omega) \rightarrow L^{1}(G, \omega) .
$$

So $\operatorname{ker}(\tilde{\pi})$ is a dual Banach $L^{1}(G, \omega)$-bimodule.

Consider the continuous derivation $D: L^{1}(G, \omega) \rightarrow \operatorname{ker}(\tilde{\pi})$ defined by

$$
D(f)=f \cdot \delta_{(e, e)}-\delta_{(e, e)} \cdot f \quad \forall f \in L^{1}(G, \omega) .
$$

Since $L^{1}(G, \omega)$ is boundedly approximately amenable, $D$ is boundedly approximately inner. This ensures that there is a net $\left(u_{i}\right)$ of elements of $M(G \times G, \omega \times \omega)$ and a constant $C>0$ such that

$$
\tilde{\pi}\left(u_{i}\right)=\delta_{e}, \quad\left\|f \cdot u_{i}-u_{i} \cdot f\right\| \leq C\|f\| \quad \text { and } \quad\left\|f \cdot u_{i}-u_{i} \cdot f\right\| \rightarrow 0,
$$

for all $f \in L^{1}(G, \omega)$. Let $\left(e_{\alpha}\right)$ be a quasi-central approximate identity for $L^{1}(G)$ satisfying (4.1). Define $U_{(i, \alpha)}=e_{\alpha} \cdot u_{i} \cdot e_{\alpha}$. Then $U_{(i, \alpha)} \in L^{1}(G \times G, \omega \times \omega)$ and $\pi\left(U_{(i, \alpha)}\right)=e_{\alpha} * e_{\alpha}$ for each $i$. By Lemma 4.1,

$$
\lim _{\alpha}\left(e_{\alpha} \cdot u_{i} \cdot e_{\alpha}-e_{\alpha} \circ u_{i} \circ e_{\alpha}\right)=0 .
$$


For each $i$, we may choose $\alpha(i)$ such that

$$
\left\|e_{\alpha} \cdot u_{i} \cdot e_{\alpha}-e_{\alpha} \circ u_{i} \circ e_{\alpha}\right\| \leq 1 \quad \forall \alpha \geq \alpha(i) .
$$

If $f \in L^{1}(G, \omega)$, then

$$
\begin{aligned}
f \cdot U_{(i, \alpha)}-U_{(i, \alpha)} \cdot f=f & \cdot\left(e_{\alpha} \cdot u_{i} \cdot e_{\alpha}-e_{\alpha} \circ u_{i} \circ e_{\alpha}\right) \\
& -\left(e_{\alpha} \cdot u_{i} \cdot e_{\alpha}-e_{\alpha} \circ u_{i} \circ e_{\alpha}\right) \cdot f \\
& +f \cdot\left(e_{\alpha} \circ u_{i} \circ e_{\alpha}\right)-\left(e_{\alpha} \circ u_{i} \circ e_{\alpha}\right) \cdot f .
\end{aligned}
$$

Also, by the mixed commutative laws,

$$
f \cdot\left(e_{\alpha} \circ u_{i} \circ e_{\alpha}\right)=e_{\alpha} \circ\left(f \cdot u_{i}\right) \circ e_{\alpha}
$$

and

$$
\left(e_{\alpha} \circ u_{i} \circ e_{\alpha}\right) \cdot f=e_{\alpha} \circ\left(u_{i} \cdot f\right) \circ e_{\alpha} .
$$

It follows that

$$
\begin{aligned}
\left\|f \cdot U_{(i, \alpha)}-U_{(i, \alpha)} \cdot f\right\|_{\omega \times \omega} & \leq 2\|f\|_{\omega}+\left\|e_{\alpha} \circ\left(f \cdot u_{i}-u_{i} \cdot f\right) \circ e_{\alpha}\right\| \\
& \leq\left(2+c^{2} C\right)\|f\|,
\end{aligned}
$$

for all $f \in L^{1}(G, \omega)$ and $\alpha \geq \alpha(i)$, where $c>0$ is a bound for $\left\|e_{\alpha}\right\|_{\omega}$.

Finally, a standard argument together with (4.2), (4.3) and (4.4) shows that there is a subnet $\left(U_{j}\right)$ of $\left(U_{(i, \alpha)}\right)_{\alpha \geq \alpha(i)}$ such that $\left(U_{j}\right)$ is a multiplier-bounded approximate diagonal for $L^{1}(G, \omega)$ and $\left(\pi\left(U_{j}\right)\right)$ is a bounded approximate identity for $L^{1}(G, \omega)$. Therefore $L^{1}(G, \omega)$ is boundedly approximately contractible by Proposition 3.1 .

\section{Beurling algebras on IN groups}

It is shown in [7] that if $\omega$ is bounded away from 0 and $L^{1}(G, \omega)$ is approximately amenable, then $G$ is amenable. It has been conjectured that this result still holds even if $\omega$ is not assumed to be bounded below away from 0 . In this section we show that the conjecture is true for a class of groups that includes the IN groups.

We recall that a locally compact group $G$ is $I N$ if there is a compact neighborhood of the identity $e$ in $G$ that is invariant under all inner automorphisms of $G$. The group $G$ is an $[I N]_{B}$ group (or $G \in[I N]_{B}$ ) for a given subset $B$ of $G$, if there is a compact neighborhood $N_{B}$ of $e$ in $G$ such that $t N_{B} t^{-1}=N_{B}$ for all $t \in B$ [13].

THEOREM 5.1. Suppose that $G \in[I N]_{B}$ for each finite subset $B$ of $G$. Suppose that there is a neighborhood $U$ of $e$ that is invariant under inner automorphisms implemented by elements of $G$ and $K=\inf \{\omega(s): s \in U\}>0 . \quad$ If $L^{1}(G, \omega)$ is approximately amenable, then $G$ is amenable.

PROOF. We aim to prove that there is a net $\left(v_{\beta}\right) \subset L^{1}(G \times G)^{+}$satisfying the conditions $\left\|v_{\beta}\right\|_{1}=\left\langle v_{\beta}, 1\right\rangle=1$ for all $\beta$, and

$$
\delta_{t} \cdot v_{\beta}-v_{\beta} \cdot \delta_{t} \stackrel{\beta}{\longrightarrow} 0 \quad \forall t \in G
$$


in the norm topology of $L^{1}(G \times G)$. Once this is proved, one may construct a right invariant mean on $L^{\infty}(G)$ in a standard way. Let $u_{\beta}(x)=\int v_{\beta}(\xi, x) d \xi$ for all $x \in G$; then $u_{\beta} \in L^{1}(G)$ and a weak* cluster point of $\left(u_{\beta}\right)$ in $L^{\infty}(G)^{*}$ is a right invariant mean on $L^{\infty}(G)$. Thus $G$ is amenable.

To find the above net $\left(v_{\beta}\right)$, it suffices to show that, for each finite set $B \subset G$ and $\varepsilon>0$, there exists $v \in L^{1}(G \times G)^{+}$such that $\left\|\delta_{t} \cdot v-v \cdot \delta_{t}\right\|_{1}<\varepsilon$ for all $t \in B$ and $\|v\|_{1}=\langle v, 1\rangle=1$. Let $B$ be a finite set in $G$ and $\varepsilon>0$. By the hypothesis on $G$, there is a compact neighborhood $N_{B}$ of $e$ such that $t N_{B} t^{-1}=N_{B}$ for all $t \in B$. Let $h$ be the characteristic function of $N_{B}$. Then $h \in L^{1}(G) \cap L^{1}(G, \omega)$ and $\delta_{t} * h * \delta_{t^{-1}}=h$ for all $t \in B$. Since $L^{1}(G, \omega)$ has a bounded approximate identity and is approximately amenable, it is pseudo-amenable by [9, Proposition 3.2]. So there is a net $\left(m_{\alpha}\right) \subset$ $L^{1}(G \times G, \omega \times \omega)$ such that

$$
f \cdot m_{\alpha}-m_{\alpha} \cdot f \rightarrow 0 \text { and } f * \pi\left(m_{\alpha}\right) \rightarrow f,
$$

for all $f \in L^{1}(G, \omega)$, in the norm topologies of $L^{1}(G \times G, \omega \times \omega)$ and $L^{1}(G, \omega)$, respectively. If we define $\mu_{\alpha}=h \cdot m_{\alpha}$, then $\mu_{\alpha} \in L^{1}(G \times G, \omega \times \omega)$.

We now show that $\left\|\delta_{t} \cdot \mu_{\alpha}-\mu_{\alpha} \cdot \delta_{t}\right\|_{\omega \times \omega} \rightarrow 0$ for all $t \in B$. In fact, if $t \in B$, then

$$
\begin{aligned}
\delta_{t} \cdot \mu_{\alpha}-\mu_{\alpha} \cdot \delta_{t}= & \left(\delta_{t} * h\right) \cdot \mu_{\alpha}-\mu_{\alpha} \cdot\left(\delta_{t} * h\right) \\
& \quad+m_{\alpha} \cdot\left(\delta_{t} * h * \delta_{t^{-1}}-h\right) \cdot \delta_{t}+\left(m_{\alpha} \cdot h-h \cdot m_{\alpha}\right) \cdot \delta_{t} \\
= & \left(\left(\delta_{t} * h\right) \cdot \mu_{\alpha}-\mu_{\alpha} \cdot\left(\delta_{t} * h\right)\right)+\left(m_{\alpha} \cdot h-h \cdot m_{\alpha}\right) \cdot \delta_{t} .
\end{aligned}
$$

Since $\delta_{t} * h, h \in L^{1}(G, \omega)$, it follows immediately that

$$
\lim _{\alpha}\left\|\delta_{t} \cdot \mu_{\alpha}-\mu_{\alpha} \cdot \delta_{t}\right\|_{\omega \times \omega}=0
$$

for all $t \in B$. Without loss of generality, we may assume that the set $U$ described in the hypothesis on $G$ is a closed subset of $G$. Let

$$
V=\{(x, y) \in G \times G: y x \in U\} .
$$

Then its characteristic function $\chi_{V}$ is measurable, and satisfies $\chi_{V}(x, y)=\chi_{U}(y x)$ for all $x, y \in G$.

For each $\alpha$, define $n_{\alpha}=\chi_{V} \mu_{\alpha}$ to be the pointwise product of $\chi_{V}$ and $\mu_{\alpha}$. We show that $n_{\alpha} \in L^{1}(G \times G)$ and that there exists $\alpha_{0}$ such that, if $\alpha>\alpha_{0}$, then

$$
\frac{1}{2} \lambda\left(U \cap N_{B}\right) \leq\left\|n_{\alpha}\right\|_{1} \leq \frac{1}{K}\left\|\mu_{\alpha}\right\|_{\omega \times \omega}
$$

(recall that $\lambda$ denotes the left invariant Haar measure on $G$ ). In fact,

$$
\begin{aligned}
\left\|n_{\alpha}\right\|_{1} & =\int_{G \times G} \chi_{U}(y x)\left|\mu_{\alpha}(x, y)\right| d x d y \\
& \leq \frac{1}{K} \int_{G \times G} \omega(y x)\left|\mu_{\alpha}(x, y)\right| d x d y \leq \frac{1}{K}\left\|\mu_{\alpha}\right\|_{\omega \times \omega} .
\end{aligned}
$$


On the other hand $\chi_{U}\left(x^{-1} y x\right)=\chi_{U}(y)$ for all $x, y \in G$, since $U$ is invariant under inner automorphisms, and $\chi_{U} \in L^{\infty}\left(G, \omega^{-1}\right)$ since $K>0$. Thus

$$
\begin{aligned}
\left\|n_{\alpha}\right\|_{1} & =\int_{G \times G} \chi_{U}(y x)\left|\mu_{\alpha}(x, y)\right| d x d y \\
& =\int_{G \times G} \chi_{U}\left(x^{-1} y x\right)\left|\mu_{\alpha}\left(x, x^{-1} y\right)\right| d x d y \\
& =\int_{G \times G} \chi_{U}(y)\left|\mu_{\alpha}\left(x, x^{-1} y\right)\right| d x d y \geq\left|\left\langle\pi\left(\mu_{\alpha}\right), \chi_{U}\right\rangle\right| .
\end{aligned}
$$

Since $\pi\left(\mu_{\alpha}\right)=h * \pi\left(m_{\alpha}\right) \stackrel{\alpha}{\longrightarrow} h$ in $L^{1}(G, \omega)$, we deduce that

$$
\left\langle\pi\left(\mu_{\alpha}\right), \chi_{U}\right\rangle \stackrel{\alpha}{\longrightarrow}\left\langle h, \chi_{U}\right\rangle=\int_{G} \chi_{U \cap N_{B}}(x) d x=\lambda\left(U \cap N_{B}\right)>0 .
$$

Thus, there exists $\alpha_{0}$ such that $\left\|n_{\alpha}\right\|_{1}>\frac{1}{2} \lambda\left(U \cap N_{B}\right)$ for all $\alpha>\alpha_{0}$. Therefore (5.1) holds when $\alpha>\alpha_{0}$.

Moreover, for $\lambda$-almost every $t \in G$,

$$
\begin{aligned}
\left\|\delta_{t} \cdot n_{\alpha}-n_{\alpha} \cdot \delta_{t}\right\|_{1} & =\int_{G \times G} \chi_{U}\left(y t^{-1} x\right)\left|\mu_{\alpha}\left(t^{-1} x, y\right)-\mu_{\alpha}\left(x, y t^{-1}\right)\right| d x d y \\
& \leq \frac{\omega\left(t^{-1}\right)}{K} \int \omega(x) \omega(y)\left|\mu_{\alpha}\left(t^{-1} x, y\right)-\mu_{\alpha}\left(x, y t^{-1}\right)\right| d x d y \\
& =\frac{\omega\left(t^{-1}\right)}{K}\left\|\delta_{t} \cdot \mu_{\alpha}-\mu_{\alpha} \cdot \delta_{t}\right\|_{\omega \times \omega} .
\end{aligned}
$$

This implies that $\lim _{\alpha}\left\|\delta_{t} \cdot n_{\alpha}-n_{\alpha} \cdot \delta_{t}\right\|_{1}=0$ for all $t \in B$.

Now let $v_{\alpha}=\left|n_{\alpha}\right| /\left\|n_{\alpha}\right\|_{1}$ and let $M=\frac{1}{2} \lambda\left(U \cap N_{B}\right)$. Clearly, $v_{\alpha} \in L^{1}(G \times G)^{+}$, $\left\|v_{\alpha}\right\|_{1}=\left\langle v_{\alpha}, 1\right\rangle=1$, and

$$
\begin{aligned}
\left\|\delta_{t} \cdot v_{\alpha}-v_{\alpha} \cdot \delta_{t}\right\|_{1} & =\frac{1}{\left\|n_{\alpha}\right\|_{1}}\left\|\delta_{t} \cdot\left|n_{\alpha}\right|-\left|n_{\alpha}\right| \cdot \delta_{t}\right\|_{1} \\
& \leq \frac{1}{\left\|n_{\alpha}\right\|_{1}}\left\|\delta_{t} \cdot n_{\alpha}-n_{\alpha} \cdot \delta_{t}\right\|_{1} \leq \frac{1}{M}\left\|\delta_{t} \cdot n_{\alpha}-n_{\alpha} \cdot \delta_{t}\right\|_{1},
\end{aligned}
$$

for $\alpha>\alpha_{0}$ and $t \in B$. Therefore

$$
\lim _{\alpha}\left\|\delta_{t} \cdot v_{\alpha}-v_{\alpha} \cdot \delta_{t}\right\|_{1}=0 \quad \forall t \in B .
$$

So, given $\varepsilon>0$, there exists $\alpha$ such that $\left\|\delta_{t} \cdot v_{\alpha}-v_{\alpha} \cdot \delta_{t}\right\|_{1}<\varepsilon$ for all $t \in B$; further, $v_{\alpha} \in L^{1}(G \times G)^{+}$and $\left\|v_{\alpha}\right\|_{1}=\left\langle v_{\alpha}, 1\right\rangle=1$. Taking $v=v_{\alpha}$, the proof is complete.

REMARK 5.2. The conditions of Theorem 5.1 are automatically fulfilled if $G$ is an IN group. If $G \in[I N]_{B}$ for each finite subset $B$ of $G$, then $G$ must be unimodular by the argument of [13, pp. 1273 and 1276]. 


\section{2n-weak amenability of Beurling algebras}

In this section we consider abelian locally compact groups $G$, written additively. Let $\omega$ be a continuous weight on $G$, and $\phi_{\omega}$ be the augmentation character on $L^{1}(G, \omega)$, that is,

$$
\phi_{\omega}(f)=\int_{G} f(x) d x \quad \forall f \in L^{1}(G, \omega) .
$$

The following is an extension of [14, Theorem 6.4].

THEOREM 6.1. Let $G$ be a locally compact abelian group, and let $\omega$ be a continuous weight function on $G$ such that the function $y \mapsto \lim _{\sup _{x}} \omega(y+x) / \omega(x)$ is bounded. Then the following conditions on $L^{1}(G, \omega)$ are equivalent.

(1) There is no nonzero continuous point-derivation at $\phi_{\omega}$.

(2) The algebra is $2 n$-weakly amenable for some $n \in \mathbb{N}$.

(3) The algebra is $2 n$-weakly amenable for all $n \in \mathbb{N}$.

Proof. First, since $G$ is abelian and hence amenable, we may assume that $\omega \geq 1$, by [16].

It is obvious that (3) implies (2). We now show that (1) implies (3) by induction on $n$. For the case where $n=1$, this has been shown in [14]. So assume that $n \geq 1$ and that $L^{1}(G, \omega)$ is $2 n$-weakly amenable. We show that it is $(2 n+2)$-weakly amenable.

Let $\left(e_{i}\right)$ be a bounded approximate identity for $L^{1}(G, \omega)$ and let $E$ be a weak* cluster point of $\left(e_{i}\right)$ in $L^{1}(G, \omega)^{* *}$. Then $E$ is an idempotent for the first Arens product $\square$ on $L^{1}(G, \omega)^{* *}$, and $f E=E f=f$ for every $f \in L^{1}(G, \omega)$. We may also write

$$
L^{1}(G, \omega)^{* *}=E \square L^{1}(G, \omega)^{* *} \oplus(I-E) L^{1}(G, \omega)^{* *} .
$$

The actions of $L^{1}(G, \omega)$ on $X_{\omega}=(I-E) L^{1}(G, \omega)^{* *}$ are trivial.

Now, using a similar argument to [5], it can be shown that $E \square L^{1}(G, \omega)^{* *}$ is isomorphic to $\operatorname{LUC}(G, 1 / \omega)^{*}$ in the category of Banach algebras. Thus, if

$$
D: L^{1}(G, \omega) \rightarrow\left(L^{1}(G, \omega)\right)^{(2 n+2)}=\left(E \square L^{1}(G, \omega)^{* *}\right)^{(2 n)} \oplus X_{\omega}^{(2 n)}
$$

is a bounded derivation, then it follows that $D=D_{1}+D_{2}$, where $D_{1}$ is a derivation into $\left(E \square L^{1}(G, \omega)^{* *}\right)^{(2 n)}$ and $D_{2}$ is a derivation into $X_{\omega}^{(2 n)}$. Hence we can assume that $D$ maps into $\operatorname{LUC}(G, 1 / \omega)^{(2 n+1)}$.

However,

$$
L U C(G, 1 / \omega)^{*}=M(G, \omega) \oplus C_{0}(G, 1 / \omega)^{\perp},
$$

where $C_{0}(G, 1 / \omega)^{\perp}$ denotes the space of all functionals in $\operatorname{LUC}(G, 1 / \omega)^{*}$ that annihilate $C_{0}(G, 1 / \omega)$. Thus

$$
L U C(G, 1 / \omega)^{(2 n+1)}=M(G, \omega)^{(2 n)} \oplus\left(C_{0}(G, 1 / \omega)^{\perp}\right)^{(2 n)} .
$$

It is shown in [14] that $C_{0}(G, 1 / \omega)^{\perp}$ is an $L^{1}(G, \omega)$ Banach bimodule, and consequently $\left(C_{0}(G, 1 / \omega)^{\perp}\right)^{(2 n)}$ is too. Hence all continuous derivations from $L^{1}(G, 1 / \omega)$ 
into $\left(C_{0}(G, 1 / \omega)^{\perp}\right)^{(2 n)}$ vanish, by [14, Theorem 4.5], and we may assume that $D$ maps into $M(G, \omega)^{(2 n)}$. However, because $L^{1}(G, \omega)$ has a bounded approximate identity, $D$ maps into $L^{1}(G, \omega) \cdot M(G, \omega)^{(2 n)}=L^{1}(G, \omega)^{(2 n)}$, and so $D=0$ by induction.

Finally, to prove that (2) implies (1), take $n \in \mathbb{N}$ such that $L^{1}(G, \omega)$ is $2 n$-weakly amenable. Then $L^{1}(G, \omega)$ is certainly 2 -weakly amenable, and the result follows by [14, Theorem 6.4$]$.

\section{Acknowledgement}

The authors would like to thank the referee for a careful reading of this paper.

\section{References}

[1] O. Y. Aristov, V. Runde and N. Spronk, 'Operator biflatness of the Fourier algebra and approximate indicators for subgroups', J. Funct. Anal. 209 (2004), 367-387.

[2] Y. Choi, F. Ghahramani and Y. Zhang, 'Approximate and pseudo-amenability of various classes of Banach algebras', J. Funct. Anal. 256 (2009), 3158-3191.

[3] H. G. Dales, F. Ghahramani and N. Grønbæk, 'Derivations into iterated duals of Banach algebras', Studia Math. 128 (1998), 19-54.

[4] H. G. Dales and A. T. M. Lau, 'The second duals of Beurling algebras', Mem. Amer. Math. Soc. 177(836) (2005).

[5] F. Ghahramani and A. T. Lau, 'Isometric isomorphisms between the second conjugate algebras of group algebras', Bull. Lond. Math. Soc. 20 (1988), 342-344.

[6] F. Ghahramani and R. J. Loy, 'Generalized notions of amenability', J. Funct. Anal. 208 (2004), 229-260.

[7] F. Ghahramani, R. J. Loy and Y. Zhang, 'Generalized notions of amenability, II', J. Funct. Anal. 254 (2008), 1776-1810.

[8] F. Ghahramani and G. Zabandan, '2-Weak amenability of a Beurling algebra and amenability of its second dual', Int. J. Pure Appl. Math. 16 (2004), 75-86.

[9] F. Ghahramani and Y. Zhang, 'Pseudo-amenable and pseudo-contractible Banach algebras', Math. Proc. Cambridge Philos. Soc. 142 (2007), 111-123.

[10] N. Grønbæk, 'Amenability of weighted convolution algebras on locally compact groups', Trans. Amer. Math. Soc. 319 (1990), 765-775.

[11] V. Losert and H. Rindler, 'Asymptotically central functions and invariant extensions of Dirac measure', in: Probability Measures on Groups VII, Lecture Notes in Mathematics, 1064 (ed. H. Heyer) (Springer, Berlin, 1984), pp. 368-378.

[12] R. Mosak, 'Central functions in group algebras', Proc. Amer. Math. Soc. 29 (1971), 613-616.

[13] T. W. Palmer, Banach Algebras and the General Theory of *-Algebras I, II (Cambridge University Press, Cambridge, 1994, 2001).

[14] E. Samei, 'Weak amenability and 2-weak amenability of Beurling algebras', J. Math. Anal. Appl. 346 (2008), 451-467.

[15] R. Stokke, 'Quasi-central bounded approximate identities in group algebras of locally compact groups', Illinois J. Math. 48 (2004), 151-170.

[16] M. C. White, 'Characters on weighted amenable groups', Bull. Lond. Math. Soc. 23 (1991), 375-380.

[17] Y. Zhang, 'Solved and unsolved problems in generalized notions of amenability for Banach algebras', in: Banach Algebras 2009, Banach Centre Publications, 91 (eds. R. J. Loy, A. Sołtysiak and V. Runde) (Polish Academy of Sciences, Institute of Mathematics, Warsaw, 2010), pp. 441-454. 
F. GHAHRAMANI, Department of Mathematics, University of Manitoba, Winnipeg, Canada R3T 2N2

e-mail: fereidou@cc.umanitoba.ca

E. SAMEI, Department of Mathematics and Statistics,

University of Saskatchewan, Saskatoon, Canada S7N 5E6

e-mail: samei@math.usask.ca

YONG ZHANG, Department of Mathematics, University of Manitoba, Winnipeg, Canada R3T 2N2

e-mail: zhangy@cc.umanitoba.ca 